\title{
Response Characteristics of Basolateral and Centromedial Neurons in the Primate Amygdala
}

\author{
Clayton P. Mosher, Prisca E. Zimmerman, and Katalin M. Gothard \\ Department of Physiology, College of Medicine, The University of Arizona, Tucson, Arizona 85724
}

\begin{abstract}
Based on cellular architecture and connectivity, the main nuclei of the primate amygdala are divided in two clusters: basolateral (BL) and centromedial (CM). These anatomical features suggest a functional division of labor among the nuclei. The BL nuclei are thought to be involved primarily in evaluating the emotional significance or context-dependent relevance of all stimuli, including social signals such as facial expressions. The CM nuclei appear to be involved in allocating attention to stimuli of high significance and in initiating situationappropriate autonomic responses. The goal of this study was to determine how this division of labor manifests in the response properties of neurons recorded from these two nuclear groups. We recorded the activity of 454 single neurons from identified nuclear sites in three monkeys trained to perform an image-viewing task. The task required orienting and attending to cues that predicted trial progression and viewing images with broadly varying emotional content. The two populations of neurons showed large overlaps in neurophysiological properties. We found, however, that CM neurons show higher firing and less regular spiking patterns than BL neurons. Furthermore, neurons in the CM nuclei were more likely to respond to task events (fixation, image on, image off), whereas neurons in the BL nuclei were more likely to respond selectively to the content of stimulus images. The overlap in the physiological properties of the CM and BL neurons suggest distributed processing across the nuclear groups. The differences, therefore, appear to be a processing bias rather than a hallmark of mutually exclusive functions.
\end{abstract}

\section{Introduction}

The mammalian amygdala plays an important role in detecting and learning the emotional significance of stimuli encountered by an organism (LeDoux, 2000; Paré et al., 2004). Within the systems-level framework of emotion, the amygdala contributes several component processes, such as stimulus appraisal, relevance detection, activation of neuroendocrine responses, and somatic motor expressions of emotion (Sander et al., 2005). Indeed the amygdala evaluates the emotional quality of facial expressions and vocalizations, and mediates several other aspects of social communication such as eye contact and the perception of personal space (Adolphs, 2010). As a manifestation of its role in assessing the relevance/significance of stimuli, the amygdala is involved in allocating attention to stimuli of importance (Holland and Gallagher, 1999; Adolphs et al., 2005; Roesch et al., 2010). Finally, a large part of autonomic and endocrine responses to stimuli of importance are elaborated in the amygdala. (Kaada, 1951; Kapp et al., 1982; Pascoe and Kapp, 1985; Reis and Ledoux, 1987; Laine et al., 2009). These diverse functions can be localized, to some extent, to the component nuclei of the amygdala.

\footnotetext{
Received June 22, 2010; revised Sept. 27, 2010; accepted Sept. 30, 2010.

This work was supported by Howard Hughes Medical Institute Grant 52005889 and a National Science Foundation graduate research fellowship to C.P.M. and National Institutes of Health Grant MH 070836 to K.M.G. We thank Kevin Spitler, Kelly Brooks, Haiyin Chen, and Robert Gibboni III for help collecting the data. We also thank Andrew Fuglevand for useful comments on this manuscript.

Correspondence should be addressed to Dr. Katalin M. Gothard, Department of Physiology, College of Medicine, The University of Arizona, 1501 North Campbell Avenue, Room 4104, Tucson, AZ 85724. E-mail: kgothard@email.arizona.edu.

DOI:10.1523/JNEUROSCI.3225-10.2010

Copyright $\odot 2010$ the authors $\quad 0270-6474 / 10 / 3016197-11 \$ 15.00 / 0$
}

Based on neural architecture and connectivity, the nuclei in the amygdala can be divided in two main groups (McDonald, 1992; Pape and Paré, 2010). The nuclei in the basolateral (BL) group (lateral, basal, and accessory basal nuclei) contain neurons of cortical type and are reciprocally connected to a broad array of cortical areas. The basolateral nuclei receive highly processed sensory information from the neocortex of temporal and parietal areas and project back to all sensory and association areas (with the exception of the frontal pole and primary motor cortex) (McDonald, 1998; Stefanacci and Amaral, 2002). The nuclei in the centromedial (CM) group (the central and medial nuclei and the anterior amygdaloid area) contain neurons of striatopallidal type and are reciprocally connected to subcortical structures, although they also receive cortically processed sensory stimuli (Amaral et al., 1992; McDonald, 1992). The centromedial nuclei receive input from three major sources: (1) subcortical structures of the diencephalon, midbrain, basal forebrain, and brainstem; (2) distinct cortical areas (e.g., the insula, olfactory cortex); (3) the basolateral nuclei of the amygdala (Krettek and Price, 1978; Amaral and Price, 1984; Amaral et al., 1992). By way of these connections, the neurons in the centromedial nuclei can participate in stimulus evaluation and also modulate the activity of autonomic centers to maintain a level of general vigilance. Indeed, electrical stimulation of the central nucleus increases attention and orienting (Gallagher et al., 1990; Kapp et al., 1994) by activating the cholinergic neurons in the basal forebrain. These neurons synchronize cortical activity and switch the cortex from slow oscillations to low-amplitude fast oscillations (Dringenberg and Vanderwolf, 1996) characteristic of attentive states. 
The existence of these two distinct processing loops converging in the amygdala suggests that the basolateral nuclei are primarily involved in evaluating the emotional content of sensory inputs, whereas the centromedial nuclei generate an enhanced level of attention and arousal necessary for improved signal detection (Davis and Whalen, 2001). Although this functional dichotomy has been challenged by experiments that showed involvement of the central nucleus in stimulus-reinforcement associations (Hall et al., 2001; Wilensky et al., 2006), it nevertheless predicts that neurons in the centromedial and basolateral nuclei should respond differentially to the appetitive and attention-demanding aspects of a task and spontaneous evaluation of stimuli. Specifically, the early, attentionrelated aspects of the task should engage primarily the neurons in the centromedial nuclei, whereas the later aspects of the task, when the monkey processes the emotional content of the image, should engage primarily the neurons in the basolateral nuclei. We tested these predictions in 454 neurons recorded from three monkeys trained to perform a simple, image-viewing task.

\section{Materials and Methods}

Surgical procedures. All surgical procedures were performed in compliance with National Institutes of Health guidelines and were approved by the Institutional Animal Care and Use Committee at the University of Arizona.

Three adult male Rhesus monkeys (Macaca mulatta) (monkeys H, T, and Q) were surgically implanted with recording chambers over the right amygdala. The implant contained three titanium pins to which a headholding ring could be attached. During recordings, the ring was secured into a rigid frame (for details, see Gothard et al., 2007). Before surgery, the monkeys were preanesthetized with ketamine $(10-15 \mathrm{mg} / \mathrm{kg}, \mathrm{i} . \mathrm{m}$.) and brought to surgical levels of anesthesia with isoflurane (1-1.5\%). The chamber was placed at stereotaxic coordinates calculated from structural magnetic resonance imaging (MRI) performed before surgery. A 6-8 $\mathrm{mm}$ diameter craniotomy, performed in the center of the chamber, was sealed with silicone elastomer (Kwik Seal; WPI). The removable seal kept the dura sterile and supple and prevented the growth of scar tissue, making the use of antibiotics, daily chamber maintenance, and dura debridement unnecessary (Spitler and Gothard, 2008).

Localization of the recording sites. To achieve precisely targeted electrode placement, each monkey underwent a presurgical structural MRI scan to localize nuclei of the amygdala. The animal's head was secured in an MRI-compatible stereotaxic apparatus (Crist Instruments) and then centered in a GE Healthcare 3T94 magnet running 11X software. A highresolution inversion-prepared spoiled gradient echo was collected, with an echo time (TE) of $3.2 \mathrm{~ms}$, repetition time (TR) of $7.6 \mathrm{~ms}$, flip angle (FA) of $20^{\circ}$, inversion time of $500 \mathrm{~ms}$, bandwidth (BW) of $31.25 \mathrm{kHz}$, a field of view (FOV) of $17 \mathrm{~cm}$, a slice width of $1 \mathrm{~mm}$, a skip of $-0.5 \mathrm{~mm}$, a matrix of $256 \times 256$, and 5 averages, with a scan time of $34 \mathrm{~min}, 44 \mathrm{~s}$. These parameters yielded $0.66 \times 0.66 \times 0.5 \mathrm{~mm}$ voxels in monkeys $\mathrm{Q}$ and $\mathrm{T}$. In monkey $\mathrm{H}$, a different set of parameters was used that yielded slices of $1 \mathrm{~mm}$ thickness. Each slice was imported into CorelDRAW (CorelDRAW Graphics Suite X5; Corel), and the boundaries of the amygdala nuclei were drawn on the image. The boundary between the BL nuclei and the CM nuclei was often visible as a thin layer of white matter, similar to a capsule that surrounds the central nucleus. The boundaries between the lateral, basal, and accessory basal nuclei were rarely visible; they were mostly inferred based on the known topography of the nuclei. From the stereotaxic coordinates calculated by this method, a recording chamber was implanted centered over the amygdala.

A second MRI scan was taken after implantation. During this scan, a slip-fitting insert containing a $4 \mathrm{~mm}$ diameter central column of vitamin $\mathrm{E}$ was placed in the chamber. The column of vitamin $\mathrm{E}$ was visualized as a high-contrast rectangular area that could be extended toward the amygdala in each slice. The axis of the vertical extension of the vitamin $\mathrm{E}$ column became a chamber-centered coordinate reference. The electrodes were always parallel with the axis of the chamber. The recording targets in each nucleus were defined as $x-y$ coordinates (relative to the
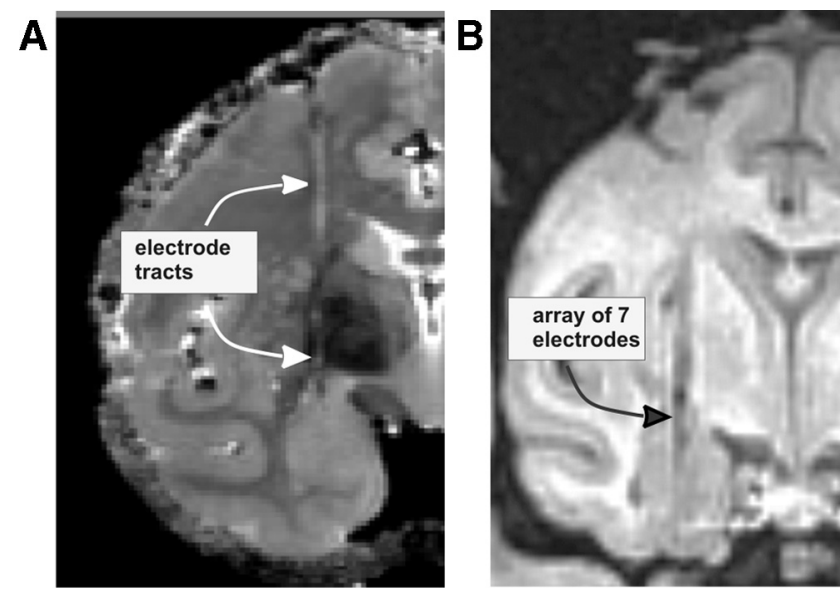

Figure 1. Localization of recording sites in the amygdala. $A$, Postmortem structural MRI scan of the brain of monkey $\mathrm{H}$ shows the electrode tracks aimed at the center of the amygdala. $\boldsymbol{B}$, In vivo MRI scan of monkey T with an array of seven Thomas electrodes placed into the amygdala.

axis of the chamber) and $z$ (or depth) coordinates relative to the dura mater. The $z$ coordinates were always more precise because the Thomas drive advances the electrodes with micron precision and because we measured at the beginning of each experiment the depth of the dura relative to the chamber. We estimate that because of the pliability of the dura, the maximal error in depth measurement was $0.5 \mathrm{~mm}$. In the mediolateral dimension, we estimated a maximum error of $1 \mathrm{~mm}$ attributable to possible discrepancies between the expected and the real boundaries separating the lateral, basal, and accessory basal nuclei. The mediolateral dimension of these nuclei rarely exceeds $2 \mathrm{~mm}$; therefore, it is unlikely that our boundary estimates were $>1 \mathrm{~mm}$ away from the real boundaries.

Postmortem structural MRI analysis of monkey $\mathrm{H}$ shows that the electrode tracks were aimed at the amygdala (Fig. $1 A$ ). In one experiment of monkey $\mathrm{T}$, the electrodes were advanced into the amygdala, detached from the drive and left in the brain. Later, the monkey was anesthetized and the array of seven electrodes visualized by a modified MRI procedure (Fig. $1 B$ ). For this scan, we used a two-dimensional gradient recalled echo run with a TE of $4.7 \mathrm{~ms}$, a TR of $50 \mathrm{~ms}$, an FA of $30^{\circ}$, a BW of 31.2, a FOV of $12 \mathrm{~cm}$, a slice width of $1 \mathrm{~mm}$, a skip of $0 \mathrm{~mm}$, a matrix of $256 \times$ 256, an Nex of 40, and a scan time of 17 min, $5 \mathrm{~s}$.

Measurement of $\mathrm{z}$-coordinate of electrode tip. At the end of each recording session, a form-fitting silicone elastomer was placed in the craniotomy, according to a method described by Spitler and Gothard (2008). When the chamber was opened, the silicone elastomer sealant was removed, and an insert was introduced in the chamber. The role of this insert was equivalent with the grid used by other neurophysiology laboratories. The insert was a sterile slip fitting stainless-steel cylinder with a $4 \mathrm{~mm}$ diameter cylindrical "tunnel" in its center. When the bottom of the insert rested on the bone margin of the craniotomy (i.e., it could not be pushed any further), the top of insert was $2-3 \mathrm{~mm}$ higher than the top of the chamber (slightly different for each monkey). At this stage, a sterile measuring cylinder was introduced into the "tunnel" of the insert, and when it rested on the dura, the point where it emerged from the insert was marked with a sterile pen. The distance between the top of the insert and the dura was determined by measuring with calipers the distance between the pen mark and the end of the measuring cylinder. Because of the use of the silicone elastomer, there was no dura overgrowth under the seal, and the dura remained pliable. This measure, therefore, was always the same. It was nevertheless needed, because we often used inserts with tunnels that were eccentric to allow recordings from multiple areas in the $X$ and $Y$ dimensions of the chamber. As the dura is sloped, the distance between the top of the insert and the dura depended on the mediolateral coordinates of the tunnel of the insert. In the next step, the $4 \mathrm{~mm}$ "head" of the Thomas drive was aligned with the axis of tunnel of the insert using the Thomas precision positioning system. The "head" fits into the tunnel and has seven sharpened cannula of $6 \mathrm{~mm}$ length at its end (at this time 
the electrodes were withdrawn into the cannulae). The tips of the cannulae were aligned with the top of the insert. The "head" with the cannulae was lowered into the tunnel to the same distance measured by the measuring cylinder. At this point, the tips of the cannulae were touching the dura. The cannulae were advanced 3-6 $\mathrm{mm}$ through the dura. The electrodes that had been withdrawn into the cannula with micron precision by the motor of the drive were then extruded into the brain. The $z$-coordinate of the electrode tip was obtained by adding $3-6 \mathrm{~mm}$ to the reading of the final electrode.

Electrodes and electrode delivery system. A custom-built, seven-channel Eckhorn drive, manufactured by Thomas Recording, was used to advance up to seven electrodes $(80-100 \mu \mathrm{m}$ shaft diameter, tungsten/platinum core, quartz glass coated) to depths of $25-35 \mathrm{~mm}$ below the dura. The electrodes were delivered via 30 ga stainless-steel sharpened cannulae that penetrated the dura and were advanced 3-6 $\mathrm{mm}$ into the cortex. The electrodes emerged from the cannulae and were advanced into the brain by precision motors that tensed or relaxed a rubber tube attached to the back of the electrode (Mountcastle et al., 1991; Eckhorn and Thomas, 1993). The electrodes were connected to a headstage amplifier (gain, 20) built into the drive and to a Lynx- 8 amplifier (Neuralynx) set to a gain of 2000 and to bandpass settings of 0.6 to $6 \mathrm{kHz}$. Neural data were digitized at $30 \mathrm{kHz}$, recorded continuously using a Power 1401 data acquisition system (Cambridge Electronic Designs), and stored on disk for off-line spike sorting.

Stimuli. The stimuli consisted of monkey faces, extracted from an extended library of digitized images of monkey facial expressions (Gothard et al., 2004) and other images that included human faces, random objects, landscapes, animals, food items, and abstract images. A total of 681 images (383 monkey faces and 298 nonfaces) were assembled in different proportions into 59 unique stimulus sets (for examples of stimuli, see Figs. 4-6). On average, a stimulus set consisted of 13 images ( 8 monkey faces, 5 nonfaces) with the smallest stimulus set having 6 images ( 6 monkey faces, 0 nonfaces) and the largest set containing 30 images ( 24 monkey faces, 6 nonfaces). All stimulus sets contained at least two monkey faces. These variations of the stimulus set prevented the monkeys from building expectations vis-á-vis the content of the images and/or habituating to a fixed experimental routine (same number of images in a set).

Recording procedures. At the beginning of a recording session, the 4 mm diameter "head" of the electrode drive was placed in the recording chamber and positioned such that the electrodes would emerge parallel with the axis of the chamber (using the Thomas precision positioning system). The electrodes were aimed at nuclei selected as targets for that recording session. The electrodes were then advanced at a speed of $30-$ $100 \mu \mathrm{m} / \mathrm{s}$ to the required depth. The depth was calculated based on the MRI slice that contained the target area. The electrodes were lowered under electrophysiological monitoring; background activity and neural signals changed at the crossing of boundaries between cortex, white matter, and gray matter at the expected depths. When the electrodes reached the dorsal border of the amygdala, the speed was reduced, and the electrodes were advanced individually by small increments until they reached the desired nucleus. When all seven electrodes registered well isolated and stable spikes, the experiment began. It was not always possible to isolate single neurons on all seven electrodes, and therefore the yield of usable single units varied from between 2 and 22 cells recorded simultaneously. The recordings were typically stable for $60 \mathrm{~min}$.

During recordings, the stimuli were presented in blocks. Each block consisted of all the images in the stimulus set used for that particular recording session. The images in a block were presented in pseudorandom succession. Each block of the same images was repeated 8-30 times, depending on the performance of the monkey. If the monkey looked outside the boundary of an image (error trial), that image was repeated at a later time. A new block was started only if the monkey looked at all images in the current block. Thus, organizing the stimuli in blocks ensured that each image was seen the same number of times.

A "trial" constituted a single image presentation. A block of trials, therefore, consisted of one presentation of each image (13 images on average). Each block was presented 8-30 times. The number of images a monkey viewed during an experiment was the product of the number of images in a block (13) and the number of times a block was repeated $(8-30)$. Each trial started with a fixation. The monkeys were trained to fixate on a white square ("fix spot") that subtended 0.5 degree of visual angle (DVA). Successful fixation (maintaining gaze for $100 \mathrm{~ms}$ in an area of $1.5 \times 1.5$ DVA surrounding the fix spot) was followed by image presentation. The monkeys were then free to view the image for $3 \mathrm{~s}$, with the requirement to maintain gaze within the image boundaries. If this requirement was met for the entire duration of the display, the monkeys received $0.5-1 \mathrm{ml}$ yogurt mixed with fruit juice. Each image was followed by a $3 \mathrm{~s}$ intertrial interval. When the monkey failed to fixate or looked outside the boundary of the image, the trial was terminated, reward was withheld, and a $2 \mathrm{~s} \mathrm{time-out} \mathrm{period} \mathrm{ensued.} \mathrm{Incorrect} \mathrm{trials} \mathrm{were} \mathrm{repeated}$ at a later time. Eye movements were sampled ( $120 \mathrm{~Hz}$ sampling, $0.3 \mathrm{DVA}$ spatial resolution) using an infrared eye tracker (ISCAN) that connected to a CORTEX experimental control system (National Institutes of Mental Health-supported freeware, http://www.cortex.salk.edu).

Data analysis. We used a template matching algorithm for off-line spike sorting (Spike 2; Cambridge Electronics Design). All other analyses were performed using MATLAB 7.9 software (MathWorks).

The basic firing properties of the recorded neurons were assessed by the interspike interval (ISI) distribution, mean firing rate, and coefficient of variation of the interspike intervals calculated for the entire recording session or for various epochs of each trial. Interspike interval histograms were computed using $20 \mathrm{~ms}$ time bins spanning $0-500 \mathrm{~ms}$. Mean firing rate was calculated as the inverse of the mean of all ISIs. The coefficient of variation was calculated as the ISI standard deviation divided by the ISI mean.

The task or image-related properties of the recorded neurons were assessed by peristimulus time histograms (PSTHs). PSTHs were computed in windows centered both on fix-spot and image presentations ( -300 to $300 \mathrm{~ms}$ around fix spot on; -2000 to $5000 \mathrm{~ms}$ relative to image on). Spikes were convolved with a $100 \mathrm{~ms}$ Gaussian window and averaged across trials to yield PSTHs expressed in units of mean firing rate. PSTHs were $z$-score normalized by subtracting the mean firing rate and dividing by the standard deviation of the baseline firing rate (firing rate during a $1 \mathrm{~s}$ period of the intertrial interval preceding the fix spot by $500 \mathrm{~ms}$ ).

For comparing the proportion of responses of a particular type between the two nuclear groups, $\chi^{2}$ tests were used. For comparison between mean values (e.g., firing rate, selectivity), two-sample $t$ tests were used.

Classification criteria of neural responses. The spike trains were aligned to the following task events: "fix spot on" is the time of display of the fixation icon; "image on" and "image off" are the times of the display and removal of the image from the monitor, respectively. Taskdependent firing properties were grouped in phasic (excitatory or inhibitory) and tonic (excitatory or inhibitory) response types (Fig. 2) as described below.

Phasic responses were identified as a brief increase in firing rate that followed one of the following task events: fix-spot presentation, image presentation (any image), or the end of the trial. In a time window around each marker ( $80-275 \mathrm{~ms}$ after fix spot on; $80-450 \mathrm{~ms}$ after image on; 80-275 ms after image off), the peak and dip times were identified as the time points when the mean firing rate achieved its maximum or minimum values, respectively. The peak/dip firing rate was measured as the mean firing rate in a $100 \mathrm{~ms}$ bin centered on this time. This set of firing rates for an individual neuron across repeated trials was then compared to relevant baseline periods. If a unit was classified as phasic responsive for any task event, the response latency was quantified as the peak/dip time.

To determine whether neurons responded to the fix spot, the mean baseline firing rate was computed for each trial in six $50 \mathrm{~ms}$ bins preceding fix spot on by $300 \mathrm{~ms}$. The baseline firing rates were then compared to the peak/dip firing rates (computed as a mean firing rate for each trial during the peak/dip time period) using a two-sample $t$ test. If the $t$ value was positive and corresponded to $p<0.01$, the response was classified as fix-spot-on phasic excitatory; if the $t$ value was negative and $p<0.01$, the response was classified as fix-spot-on phasic inhibitory.

To determine whether neurons responded to image onset, the mean baseline firing rate was calculated in $100 \mathrm{~ms}$ bins spanning two periods: a 

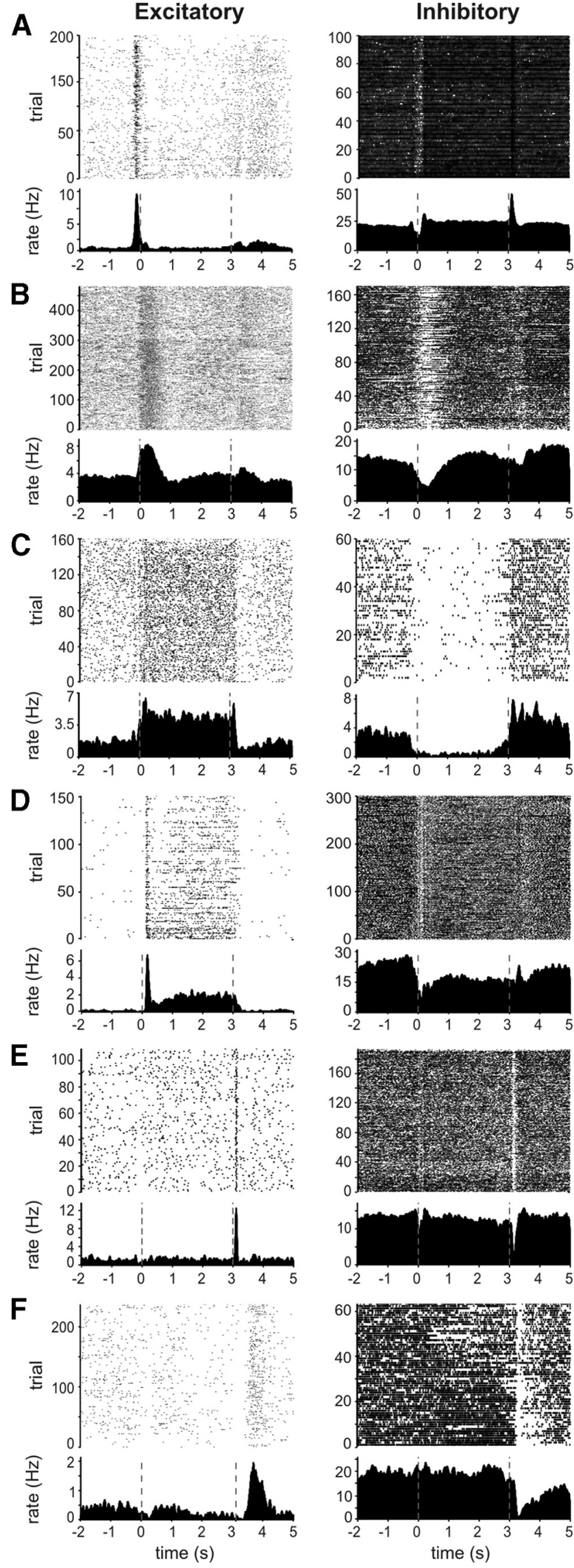

Figure 2. Classification of neural response types observed in the amygdala during an imageviewing task. Each row contains two example neurons that show an excitatory (left) and inhibitory pre-fix-spot baseline ( -1500 to $-500 \mathrm{~ms}$ preceding fix spot on) and an image baseline (500-1500 ms after image on). This second baseline was necessary to ascertain whether the response was indeed phasic and diminished (for excitatory responses) or increased (for inhibitory responses) relative to the peak/dip period. Separate two-sample $t$ tests were used to compare the peak/dip firing rate to the binned firing rate during the pre-fix-spot and image baselines. If both of these $t$ values were positive and corresponded to $p<0.01$, the response was classified as image-on phasic excitatory; if they were negative, the response was classified as image-on phasic inhibitory.

For each image-off marker, the mean baseline firing rate was calculated in $100 \mathrm{~ms}$ bins also spanning two baseline periods: a pre-image-off baseline ( -1500 to 0 preceding image off) and an intertrial-interval baseline (500-1500 ms after image off). Separate two-sample $t$ tests were used to compare the peak/dip firing rate to the binned firing rate during the pre-image-off and intertrial-interval baselines. If both of these $t$ values were positive and corresponded to $p<0.01$, the unit was classified as end-trial phasic excitatory; if they were negative, the response was classified as end-trial phasic inhibitory.

Tonic responses were identified as a sustained increase or decrease in firing rate that occurred during image presentation. For each correctly performed trial, the mean firing rate was computed in $100 \mathrm{~ms}$ bins that spanned from $500 \mathrm{~ms}$ after the image appeared to the end of image display (at $3000 \mathrm{~ms}$ ). These binned firing rate values were then pooled for all image presentations and a two-sample $t$ test was used to compare the firing rates between the image and baseline periods. If the $t$ value corresponded to $p<0.01$, the unit was classified as "image tonic" (for positive $t$ values the response was excitatory, for negative values it was inhibitory). Figure $2 C$ shows examples of tonic excitatory and tonic inhibitory responses to the images.

All responses classified as fix spot on, image on, or end-trial phasic excitatory were in agreement with the qualitative observations of the authors.

Measures of image selectivity. Selectivity was computed only for neurons that were monitored for at least eight repetitions of the stimulus set (using only presentations in which the monkey maintained its gaze within the image boundary for the required $3 \mathrm{~s}$ ).

For each cell, an average image-selectivity score was computed, using the mean firing rate, during two time windows following image on: a phasic window ( 80 to $300 \mathrm{~ms}$ ) and a tonic window (500 to $3000 \mathrm{~ms}$ ). Phasic and tonic selectivity were measured across all images in a stimulus

(right) response to the same task event. For each neuron, the rasters (top) and PSTHs (bottom) are aligned to the display and removal of the stimulus image (indicated by dotted lines at 0 and $3 \mathrm{~s})$. Image display was preceded by a $100 \mathrm{~ms}$ fixation period. Reward was delivered immediately after image offset. $A$, Fix-spot responses. Both neurons in this row show a phasic change in firing rate immediately following fix-spot display. The fix-spot neurons in the left and right panels were recorded from the central and medial nuclei, respectively. $\boldsymbol{B}$, Image-on responses. Both neurons show a phasic change of firing rate following image display and return to baseline firing within 1 s after image display. These neurons were recorded from the accessory basal (left) and central (right) nuclei. $C$, Tonic image-related responses. The increase and decrease of firing rate in both neurons lasted for the entire duration of image presentation. These neurons were recorded from the accessory basal (left) and central (right) nuclei. $\boldsymbol{D}$, Phasic-tonic imagerelated responses. These neurons showed an initial phasic change of firing rate followed by a tonic change of firing rate in the same direction (excitatory or inhibitory) but of smaller amplitude. Typically these neurons were highly selective. Both phasic-tonic image-responsive neurons shown here were recorded from the basal nucleus. $\boldsymbol{E}$, End-trial/image-off responses. A phasic change of firing rate in these neurons occurred $110-150 \mathrm{~ms}$ after the stimulus image was removed from the monitor. These neurons were recorded from the central (left) and accessory basal (right) nuclei. $\boldsymbol{F}$, End-trial/reward responses. Compared to the image-off responses, the change of firing rate in these neurons occurred later ( $\sim 200 \mathrm{~ms}$ ) and lasted longer, extending 1 s into the $3 \mathrm{~s}$ intertrial interval and overlapping in time with the delivery of reward. The excitatory (left) and inhibitory (right) end-trial/reward related responses were recorded from neurons in the central and medial nuclei respectively. As shown here, the majority of neurons respond to more than one task event; e.g., the inhibitory fix-spot response ( $A$, right) is combined with an excitatory image-off response; the phasic inhibitory end-trial response ( $\boldsymbol{E}$, right) is preceded by the phasic inhibitory image-on response. 
Table 1. Nuclear localization of cells recorded from each monkey, Q, T, and H

\begin{tabular}{lrrrr}
\hline Nucleus & \multicolumn{1}{c}{$\mathrm{T}$} & $\mathrm{H}$ & Total \\
\hline Lateral & 21 & 5 & 0 & 26 \\
Basal & 10 & 12 & 39 & 61 \\
Accessory basal & 33 & 6 & 82 & 121 \\
Central & 103 & 44 & 28 & 175 \\
Medial & 16 & 4 & 31 & 51 \\
Anterior amygdaloid area & 0 & 0 & 20 & 20 \\
Total & 183 & 71 & 200 & 454
\end{tabular}

set by computing a nonparametric one-way ANOVA test statistic (the Kruskal-Wallis $H$ ratio). The $H$ ratio was normalized for stimulus set size and number of repetitions. Selectivity scores are therefore ANOVA ratios that compare the mean variability in firing rate between all the images in the stimulus set to the trial-by-trial variability in firing rate when the same image is presented multiple times. A neuron was classified as selective if the $H$ ratio was significant $(p<0.05)$ in at least one time window (phasic or tonic).

In addition to the phasic and tonic selectivity, a millisecond-bymillisecond selectivity score was calculated on the Gaussian-convolved spike train using the same nonparametric test statistic. Here, the ANOVA ratio compares the firing rate at every $1 \mathrm{~ms}$ bin (rather than comparing the average firing rates in a predefined window) and has the advantage of resolving any time-dependent properties, such as when the earliest time bin of selectivity emerges.

Neurons were characterized as being selective to monkey faces, other images, or both categories by computing peristimulus time histograms for each image in the stimulus set. If a cell fired significantly with a tonic or phasic response to at least one image in either category, it was deemed responsive to that category of stimuli.

\section{Results}

A total of 454 neurons were recorded from three monkeys in 71 recording sessions $(32,25$, and 14 sessions for monkeys $\mathrm{H}, \mathrm{Q}$, and $\mathrm{T}$, respectively) from the following nuclei of the amygdala: lateral (L), basal (B), accessory basal (AB), central (C), and medial (M) nuclei, as well as the anterior amygdaloid area (AAA) (Table 1). Neurons from the lateral, basal, and accessory basal nuclei were pooled in the BL group, whereas neurons from the central and medial nuclei and the anterior amygdaloid area were pooled into the CM group. Of the seven electrodes, three ( $\pm 0.8 \mathrm{SD}$ ) electrodes yielded $6.3( \pm 1.2 \mathrm{SD})$ well-isolated neurons/session. The number of well-isolated neurons recorded simultaneously ranged from 2 to 22 .

\section{Differences in general firing properties of neurons in $\mathrm{BL}$ versus $\mathrm{CM}$}

The general firing properties of BL and CM neurons were different both in terms of discharge variability and mean discharge rate. Neurons in the BL group had significantly $(p<0.001)$ lower coefficients of variation $(1.4 \pm 0.4)$ compared to the CM nuclei $(1.6 \pm 0.6)$. Likewise, the average firing rate in the CM group was significantly higher $(10.9 \pm 22 \mathrm{~Hz} ; p<0.001)$ than in the $\mathrm{BL}$ group $(5 \pm 9 \mathrm{~Hz})$. This latter observation replicates findings from the rodent amygdala, where a large proportion of the neurons in the central nucleus show sustained, high-rate activity (Dumont et al., 2002). At the individual nuclear level, the mean firing rates were as follows: $\mathrm{L}=9.8 \mathrm{~Hz} \pm 17.6 \mathrm{SD} ; \mathrm{B}=3.6 \mathrm{~Hz} \pm 4.2 \mathrm{SD}$; $\mathrm{AB}$ $=4.7 \mathrm{~Hz} \pm 8.1 \mathrm{SD} ; \mathrm{C}=12.2 \mathrm{~Hz} \pm 24.1 \mathrm{SD} ; \mathrm{M}=8.3 \mathrm{~Hz} \pm 14.7$ $\mathrm{SD} ; \mathrm{AAA}=5.5 \mathrm{~Hz} \pm 8.3 \mathrm{SD}$. A large number of cells in the lateral nucleus were virtually silent at rest but dramatically increased their firing rates in response to a preferred stimulus. These cells, previously documented in the cat amygdala (Paré and Gaudreau,
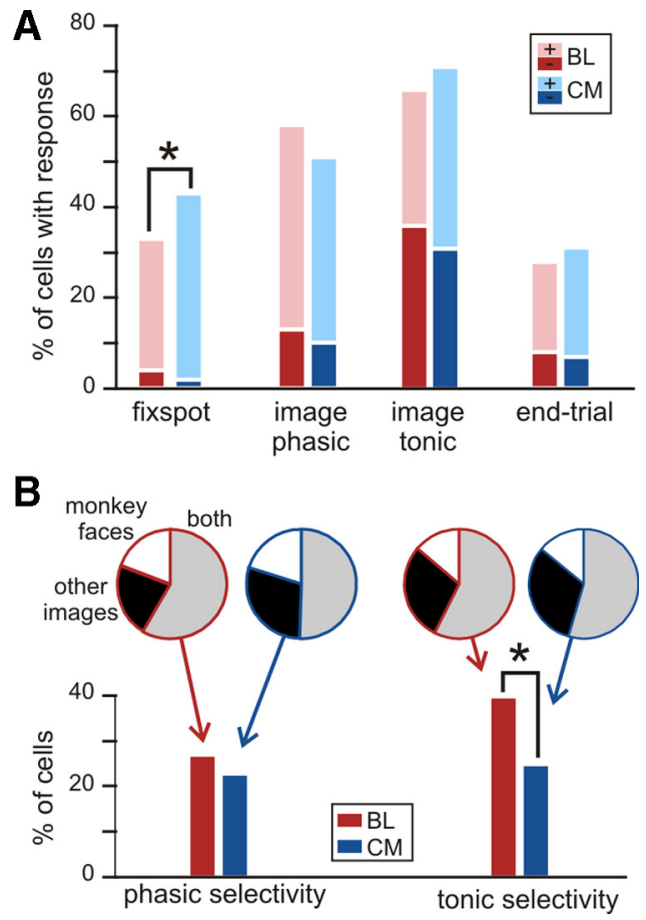

Figure 3. Summary of response properties of the population of neurons recorded from the CM and BL nuclei. $\boldsymbol{A}$, Relative proportion of neurons with task-related (fix-spot and end-trial) and image-related responses (image phasic and image tonic) in the $\mathrm{CM}$ (blue) and BL (red) groups of nuclei. Neurons with inhibitory responses are shown in a more saturated hue of color. Note that the two nuclear groups contained similar proportions of image-related neurons; however, the CM contained a significantly higher proportion of fix-spot-related neurons. The majority of fix-spot neurons were excitatory. $\boldsymbol{B}$, Relative proportion of stimulus-selective neurons in the $\mathrm{CM}$ (blue) and $\mathrm{BL}$ (red) nuclear groups. Neurons that showed selective responses in the early period of the response (phasic selectivity) were equally distributed in the CM and BL nuclei; neurons that responded selectively to images in the tonic phase of the response were significantly more numerous in the BL nuclei. The pie charts above each column represent the proportion of neurons from each category that responded selectively to monkey faces (white), to other images (black), or to both monkey faces and other images (gray). Note that the majority of cells in both nuclear groups responded to monkey faces and to other images, indicating that neurons in the amygdala are most likely to respond to multiple types of images. The least likely class of neurons in both nuclear groups are those that respond exclusively to monkey faces. ${ }^{*} p<0.05$.

1996), are discovered only if they are selective for one of the images in the stimulus set.

\section{Differences in task-related response properties}

Neural responses were grouped in several classes: fix spot on, image-on phasic, image-on tonic, and end trial (image off and reward). Of the 454 neurons, 51 neurons (11\%) showed no relationship with any aspect of the image-viewing task. Responses in either class could be phasic, tonic, or phasic and tonic (Fig. 2). Although examples for each response type were found in all sampled areas of the amygdala, the proportion of neurons that showed particular response types were unequally distributed across the two nuclear groups (Fig. 3; supplemental Table S1, available at www.jneurosci.org as supplemental material).

Fix-spot-on responses were more frequently observed in neurons recorded from CM nuclei ( $p=0.009$ ) than from BL nuclei, suggesting that orienting to the fix spot or preparing the saccade to the fix spot engages primarily the CM nuclei (Fig. $3 A$ ). Of the 454 neurons, 175 (39\% of the population) neurons showed significant fix-spot-related activity, of which 106 were recorded from the CM (supplemental Table S1, available at www.jneurosci.org as 


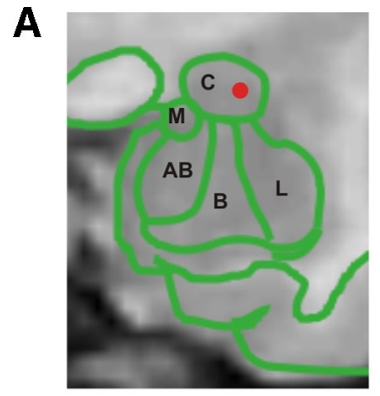

B

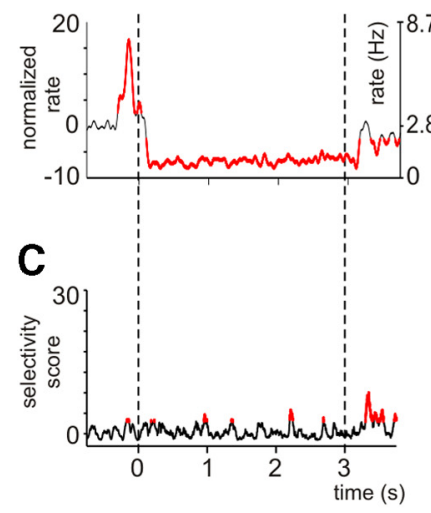

D
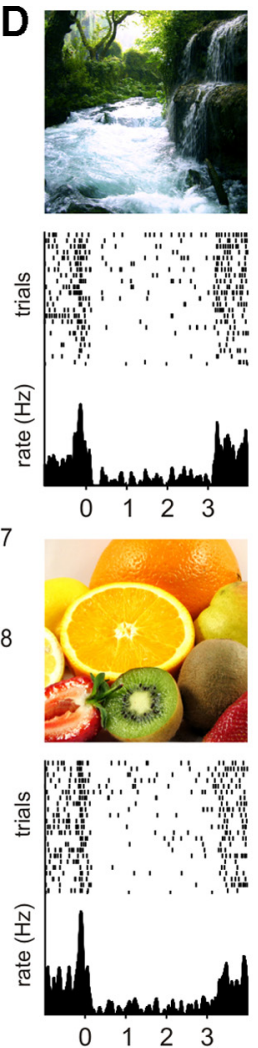
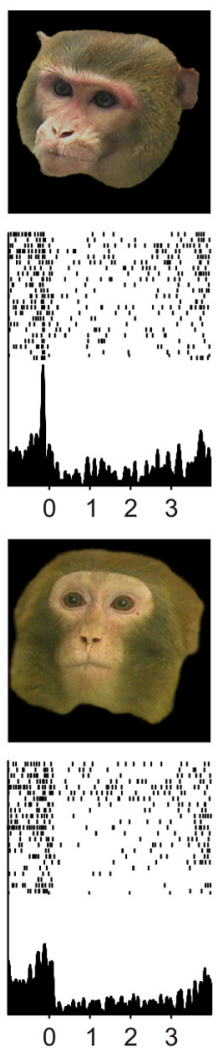
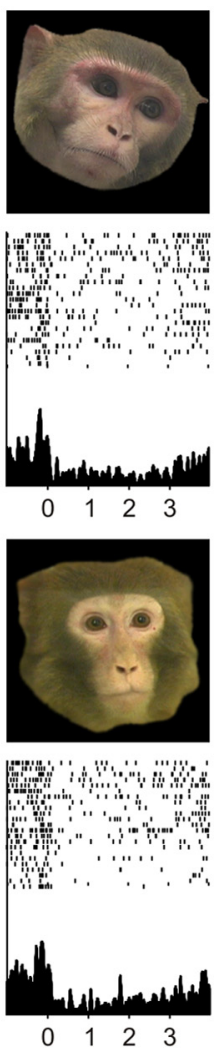
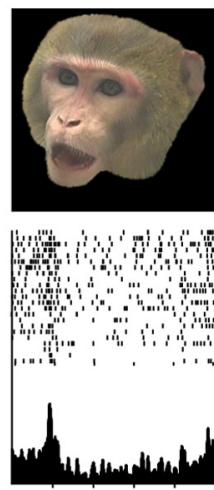

$0 \quad 123$
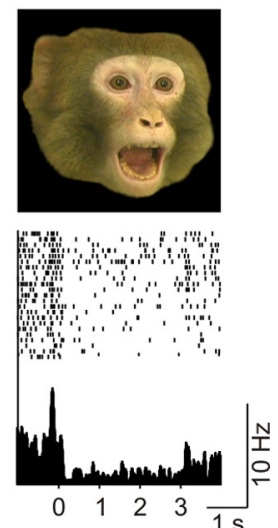

Figure 4. Example of a fix-spot-related, nonselective neuron recorded from the CM nuclei. $\boldsymbol{A}$, MRI-based reconstruction of the recording site at the center of the central nucleus. $\boldsymbol{B}$, Normalized ( $z$-score) firing rate calculated for all trials ( 0 s, image on; $3 \mathrm{~s}$, image off). The red segments indicate the time points where the firing rate was significantly $(p<0.01)$ different from the baseline firing rate (bin size, $1 \mathrm{~ms}$ ). Note that before image onset $(0 \mathrm{~s})$, the neuron showed significant increases of firing rate relative to baseline (fix-spot response), whereas after image display the firing rate was significantly reduced for the entire duration of image display (tonic inhibitory response). $C$, Normalized selectivity score ( $H$ ratio) during the trial. The red segments indicate the times when the firing pattern showed significant $(p<0.01)$ image selectivity. $D$, Rasters and PSTHs aligned to the time of image display $(0 \mathrm{~s})$. The stimuli were two nonface images and 3 facial expressions from two monkeys. Each monkey is displaying appeasing (left), neutral (middle), and aggressive (right) facial expressions.

supplemental material). These changes were always phasic and included increases or decreases of firing rate in the fix-spot window (80-275 ms after fix-spot display) relative to baseline. Examples of neurons with fix-spot-related excitatory or inhibitory responses are shown in Figure $2 A$. The majority (93\%) of fixspot-on responses were excitatory. One particular aspect of fixspot-related responses was their short latency, $154 \pm 40 \mathrm{~ms}$ SD, significantly ( $p=0.0015$ ) shorter than the latency of responses to any other aspects of the task (e.g., image on, $168 \pm 42 \mathrm{~ms}$ ). Neurons that exhibited fix-spot-related responses often showed responses to other task events (Fig. $2 \mathrm{~A}$, fix-spot-on neuron; right, image-off response).

Image-on phasic responses were equally likely in the BL and CM neurons (Fig. 3A; supplemental Table S1, available at www. jneurosci.org as supplemental material). Of the 454 neurons, 244 (54\% of the population) responded with significant increases or decreases in firing rates during the image-on phasic window $(80-$ $450 \mathrm{~ms}$ after image onset). Examples of excitatory and inhibitory phasic image-on responses are shown in Figure $2 B$. The majority (80\%) showed an excitatory response.

For image-on tonic responses, a total of 312 neurons (69\% of the population) showed sustained increases or decreases of firing rate throughout image presentation (Fig. $3 A$ ). Approximately equal fractions of neurons showed tonic excitatory and inhibitory responses in the BL and CM nuclei (supplemental Table S1, available at www.jneurosci.org as supplemental material). Examples of tonic responses are shown in Figure 2C.
End-trial responses ( 134 cells, $30 \%$ of the population) showed increases or decreases in firing rate after the image was removed from the monitor and reward delivery was signaled to the monkey (Fig. 3A). Responses that occurred within $275 \mathrm{~ms}$ after the removal of the image from the monitor were considered imageoff responses (Fig. 2E). These responses were always phasic, whether excitatory or inhibitory. Reponses with longer latencies that expanded up to $1 \mathrm{~s}$ into the intertrial interval likely corresponded to reward delivery (Fig. $2 F$ ). The majority of end-trial neurons (75\%) were excitatory (supplemental Table S1, available at www.jneurosci.org as supplemental material).

\section{Responses to multiple task events}

The majority of neurons from both nuclear groups met criteria for responding to more than one task event ( 272 cells, $60 \%$ of the recorded population). These neurons as well as the rest of the 131 (29\%) neurons that responded to a single task event were equally distributed across the two nuclear groups. Examples of neurons that responded to a single event are shown in Figure 2, $C$ (left, this neuron responded only during the image presentation), $E$ (left, this neuron responded only to image off), and $F$ (both panels, both neurons responded only to end-trial events). Examples of neurons that responded to multiple events are shown in all other panels of Figure 2.

In summary, the CM nuclei contained a larger proportion of fix-spot-responsive (43\%, or 106 of 246 ) and tonically active neu- 


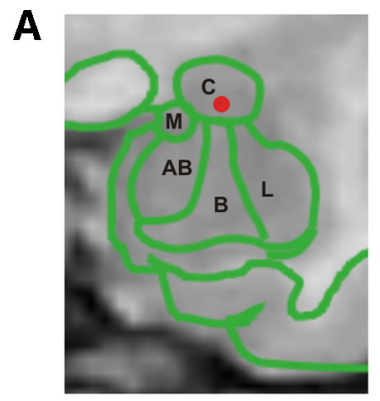

B

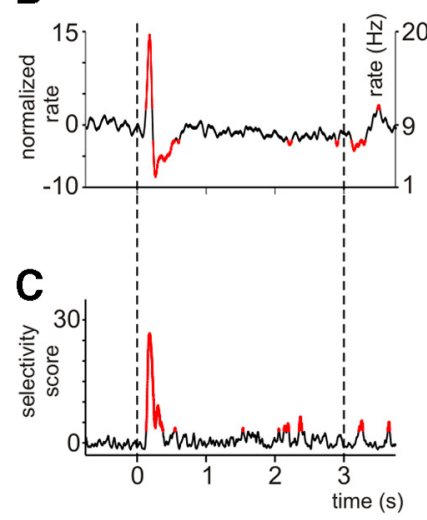

D
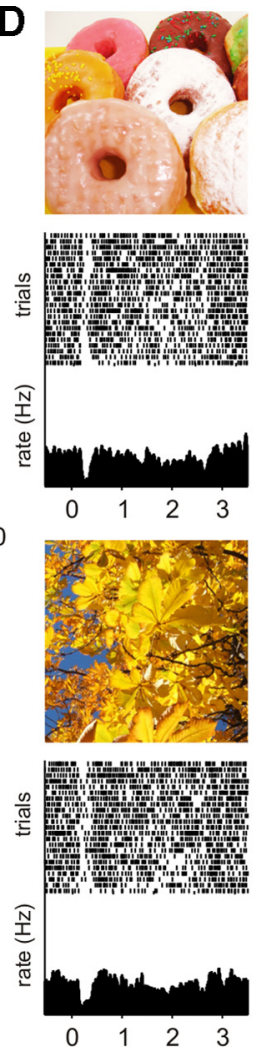
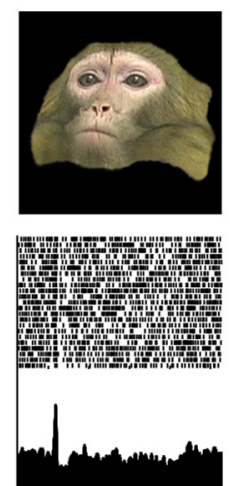

$\begin{array}{llll}0 & 1 & 2 & 3\end{array}$
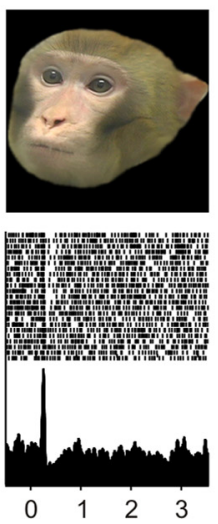
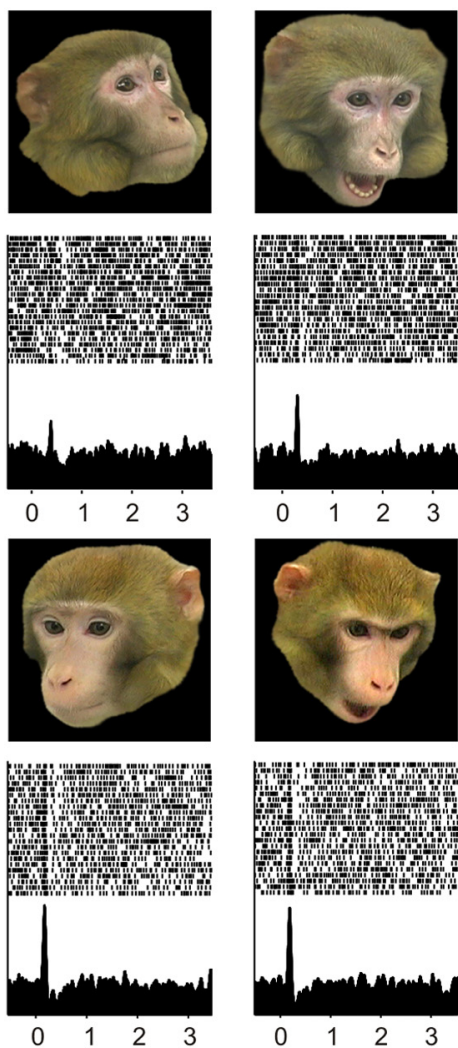

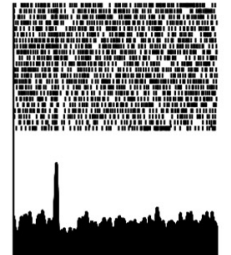

$\begin{array}{llll}0 & 1 & 2 & 3\end{array}$
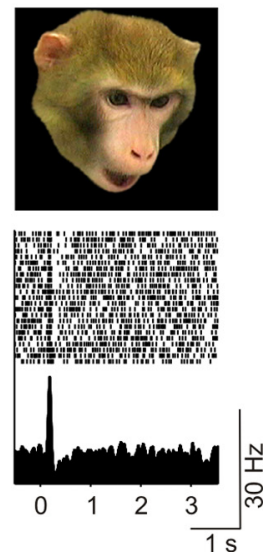

Figure 5. Image-selective, phasic responses recorded from a neuron in the central nucleus of the amygdala. $\boldsymbol{A}, \mathrm{MRI}$-based reconstruction of the recording site. $\boldsymbol{B}$, Normalized ( $\boldsymbol{Z}$-score) firing rate calculated for all trials ( $0 \mathrm{~s}$, image on; $3 \mathrm{~s}$, image off). The red segments indicate the time points where the firing rate was significantly ( $p<0.01)$ different from the baseline firing rate (bin size, 1 $\mathrm{ms})$. Note that in the earliest component of the phasic response, the firing rate was elevated compared to baseline. After this excitatory response, the firing rate showed a significant decrease and returned to baseline within $700 \mathrm{~ms}$. $C$, Normalized selectivity score ( $\mathrm{H}$ ratio) during the trial. The red segments indicate the times when the firing pattern showed significant $(p<0.01)$ image selectivity. $\boldsymbol{D}$, Neural responses to the individual images in the stimulus set, that included two nonface and six face stimuli. The faces in the top row depict the same monkey with three facial expressions: appeasing (left), neutral (middle), and threatening (right). The faces in the bottom row depict a different monkey with the same three facial expressions. Neural responses to each image are shown as rasters and PSTHs below each image. This neuron responded with a phasic inhibitory response to nonface images and with a phasic excitatory response to face images.

rons (supplemental Table S1, available at www.jneurosci.org as supplemental material).

Unequivocal, nuclear-specific classes of neurons were not observed; however, we found a small subset of nine regular-spiking neurons with firing frequencies between 2 and $5.7 \mathrm{~Hz}$ (supplemental Fig. S1, available at www.jneurosci.org as supplemental material) that were all localized to an area ventral to the central nucleus, at the boundary between the BL and CM nuclei, where intercalated neurons have been histologically identified (Ghashghaei and Barbas, 2002). Exemplars of regular-spiking neurons were found in all three monkeys. None showed task-related or image-related changes in firing pattern.

\section{Differences in image selectivity}

Stimulus discrimination or image selectivity was expressed as a selectivity score (the $H$ ratio) computed separately for the phasic and tonic segments of the neural responses during image presentation. No correlation was found between baseline firing rate and image selectivity in either nuclear group. Although highly selective neurons were found in both nuclear groups, the average selectivity score was higher in the BL nuclei.

In both the phasic and tonic segments of image-related responses, the average selectivity index in the BL nuclei was significantly higher than in the CM nuclei. The mean selectivity indices for the phasic segment were $2.2 \pm 5.6$ for $\mathrm{BL}$ and $1.2 \pm 3$ for $\mathrm{CM}$ ( $p=0.0028$ ), whereas the mean selectivity indices for the tonic segment were $2.9 \pm 5.5$ for $\mathrm{BL}$ and $1.6 \pm 3.7$ for $\mathrm{CM}(p=0.006)$. As shown in Figure $3 B$, the BL and CM nuclei contained similar proportions of phasically selective neurons (BL, 27\%; CM, 23\%; $p=0.37)$, whereas the tonically selective neurons were relatively more numerous in the BL nuclei (BL, $40 \%$; CM, 25\%; $p=0.002)$. This finding, together with the observation that the CM nuclei contained a larger number of tonically active neurons, indicates that the tonic or phasic response types are not predictive of selectivity.

With respect to the image types that $\mathrm{CM}$ and $\mathrm{BL}$ nuclei were selective for, we found neurons that responded (1) exclusively to monkey faces, (2) to other images (mostly nonface images; however, a few contained human or animal faces, e.g., a horse) (see Fig. 6D; supplemental Fig S2, available at www.jneurosci.org as supplemental material), and (3) to a subset of monkey faces and other images (see Figs. 5, 6). The proportion of phasically responsive neurons that responded exclusively to monkey faces were 19 and $20 \%$ for the BL and CM nuclei, respectively. The proportion of neurons that responded to both monkey faces and other images were 57 and 57\% in the BL and CM nuclei, respectively. Exclusively face-responsive neurons that responded tonically to the images were somewhat fewer in both nuclear groups: 15 and $14 \%$ in the BL and CM nuclei, respectively (Fig. 3B).

As characteristic of the CM nuclei, Figure 4 depicts a neuron that responds to multiple task events (phasic excitatory to fix spot and tonic inhibitory to image) but is relatively nonselective to 
A
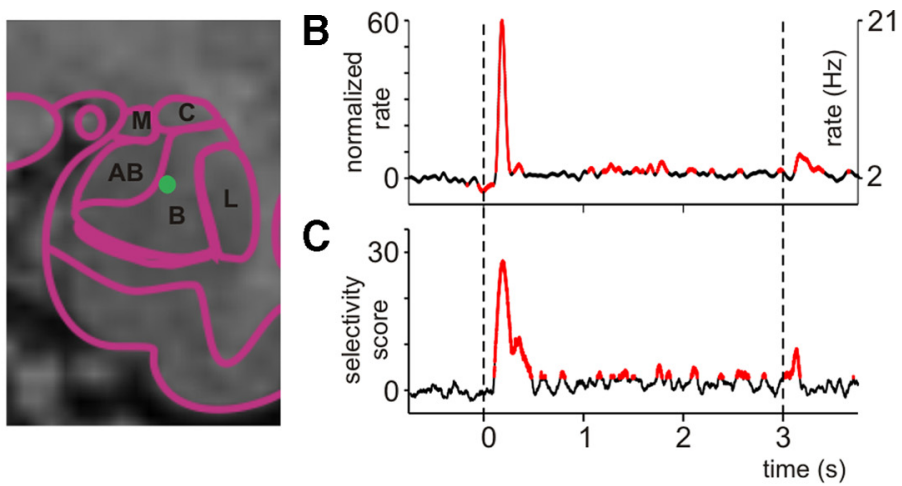

D
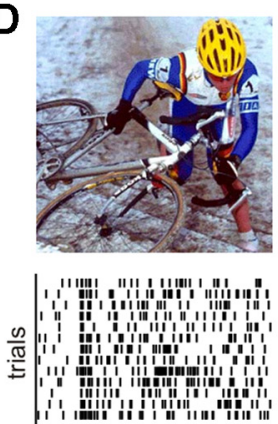

존

产
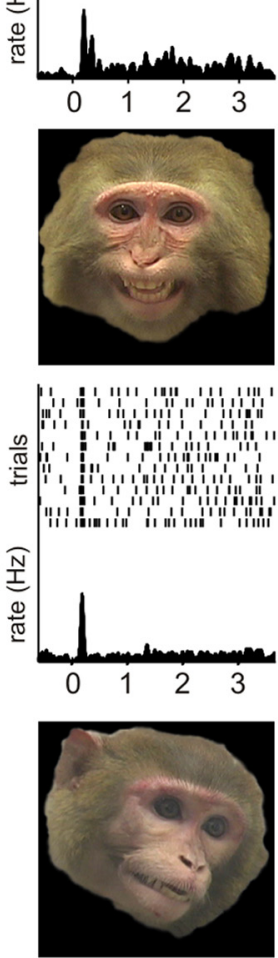

"1"

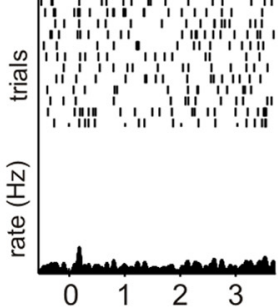

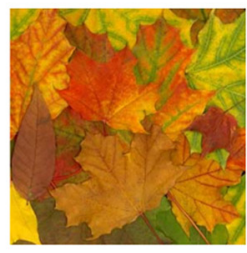
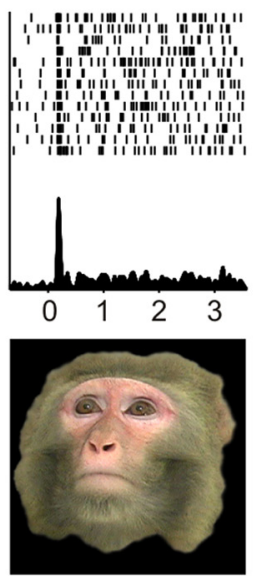

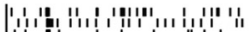
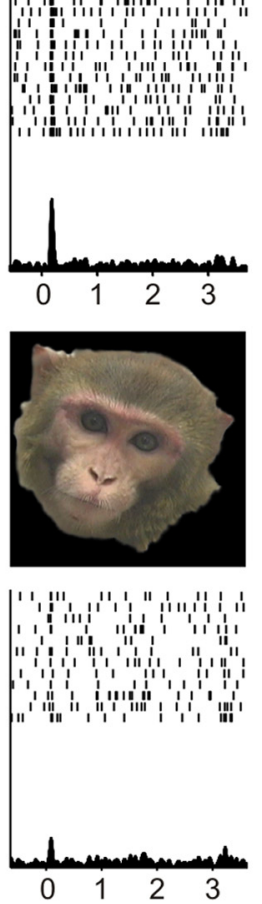
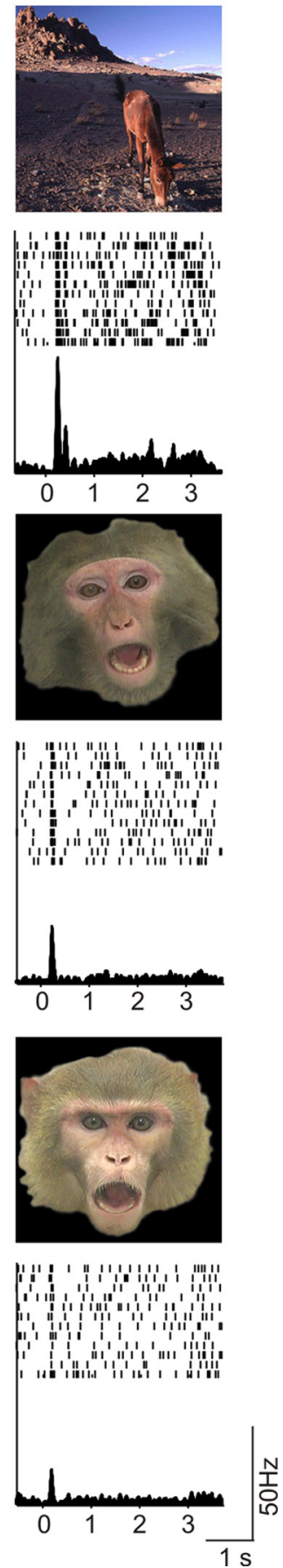

Figure 6. Example of selective, phasic-tonic responses of a neuron recorded from the basal nucleus of the amygdala. $\boldsymbol{A}$, MRI-based reconstruction of the recording site. $\boldsymbol{B}$, Normalized (z-score) firing rate of the neuron, indicating in red the bins where

images. Figure $4 B$ shows that the normalized firing rate of this neuron averaged across all images is below the baseline rate during image presentation but above baseline rate during the fixation period. The red segment of the line indicates the time points where the firing rate was significantly $(p<0.01)$ different from baseline. Figure $4 C$ indicates (in red) that selectivity was restricted to a few short periods during the tonic segment of the inhibitory response. Even though the selectivity score was significant at these points, the selectivity score of this neuron was nonetheless low (compare to selectivity indices of the neurons shown in Figs. 5, 6).

Although selectivity is, on average, higher in the BL nuclei, the CM nuclei also contained highly selective neurons. The neuron shown in Figure 5 was recorded from the central nucleus and showed phasic inhibitory responses to objects and phasic excitatory responses to monkey faces. The opposite polarity of the responses to objects and faces resulted in a high selectivity index for this neuron. Moreover, the responses to monkey faces discriminated between individuals. Figure $5 B$ shows (in red) that the normalized firing rate of this neuron increased significantly above baseline only during the phasic segment of the response. As indicated by Figure $5 C$, selectivity was likewise restricted to the phasic segment of the neural response.

An example of a highly selective neuron from the $\mathrm{BL}$ with a combined phasic and tonic response is shown in Figure 6. This neuron was face selective in the phasic segment of the response and was selective for nonface stimuli in the tonic segment.

At the population level, neurons in both nuclear groups showed a significant change in firing rate during the fixation period (at the time when the monkey at-

$\leftarrow$

the firing rate was significantly $(p<0.01)$ different from the baseline firing rate ( $0 \mathrm{~s}$, image on; $3 \mathrm{~s}$, image off). C, Selectivity score during image presentation. The red segments indicate the times when the firing pattern showed significant $(p<$ 0.01 ) image selectivity. $D$, Neural responses to images in a stimulus set that contained three nonface images (top row) and six face images. The middle row contains three images of the same monkey displaying appeasing (left), neutral (middle), and threatening (right) facial expressions. The bottom row contains the same type of facial expressions displayed by a different monkey. Neural responses to each image are shown as rasters and the corresponding PSTHs below each image. This neuron responded with a phasic and tonic elevation of firing rate to the nonface images, but with only a phasic response to the face images. 


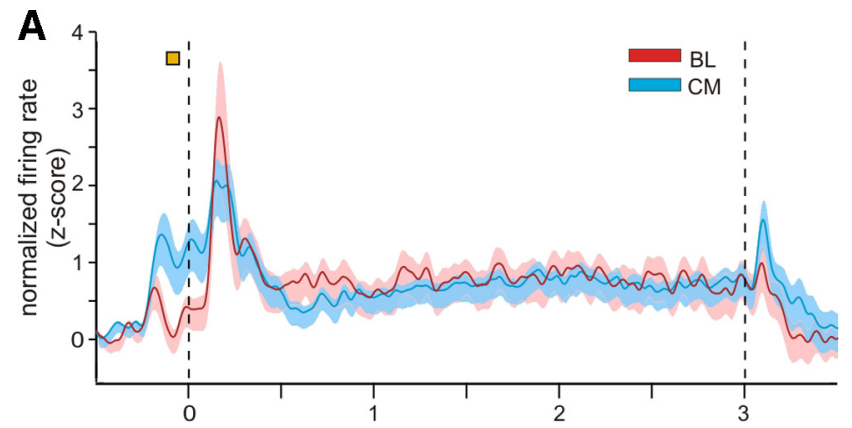

B

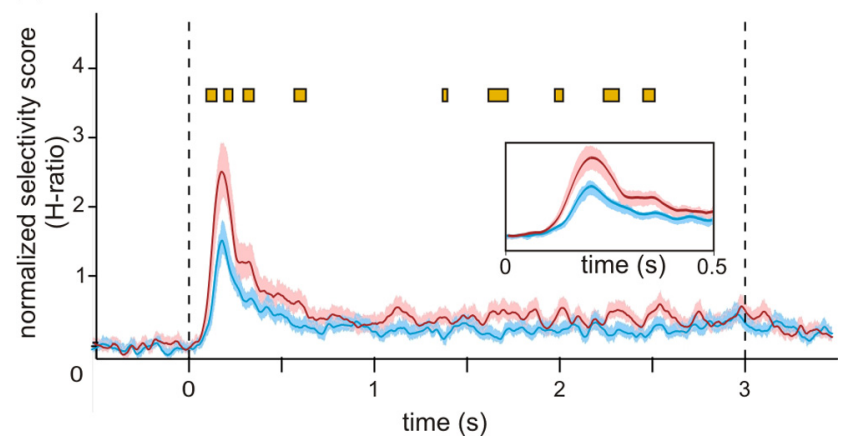

Figure 7. Population averages of firing rate changes and stimulus selectivity over time in the $\mathrm{CM}$ and $\mathrm{BL}$ nuclear groups. $\boldsymbol{A}$, Population mean \pm SEM of firing rate changes (expressed as $z$-scores) in the CM (blue) and BL (red) nuclei computed for $4.5 \mathrm{~s}$ that include the fixation period $(0.75 \mathrm{~ms}$ before 0 ) followed by image presentation (from 0 to $3 \mathrm{~s}$ ) and the end-trial period $(0.75$ $s$ after the $3 \mathrm{~s}$ marking image off). Bin size was $1 \mathrm{~ms}$ convolved with a $100 \mathrm{~ms}$ Gaussian. Significant differences $(p<0.01)$ between the population averages in adjacent bins are indicated by the yellow marks. Note that the population of $\mathrm{CM}$ neurons shows a larger increase of firing rate in the fixation period than the $B L$ population. This is because of a large fraction of fix-spot-responsive neurons in the CM nuclei. $B$, Average image selectivity (mean $H$ ratio) of the $C M$ and $B L$ populations computed for a $4 \mathrm{~s}$ period centered on image presentation ( 0 to $3 \mathrm{~s}$ ). Same bin size as in $\boldsymbol{A}$ is used. The average selectivity is higher in the BL population for several clusters of successive bins that span both the phasic ( $80-300 \mathrm{~ms}$ from image presentation) and tonic ( $500-3000 \mathrm{~ms}$ ) periods of the image presentation. The inset is an expansion of the 0 to 500 ms period after image presentation.

tended to the fix spot) and also during image presentation (when the monkey actively scanned the image and might have evaluated the emotional content of the image). The averaged firing rate across the two populations of neurons from the CM and BL nuclei showed significant differences only during the fixation period, with larger increases from baseline in the CM nuclei (Fig. $7 A$ ). In contrast, the average selectivity score for the two populations of neurons was moderately but significantly higher for the entire population of BL neurons at multiple time points during image presentation spanning both the phasic and the tonic segments of the response (Fig. $7 B$ ).

\section{Discussion}

The goals of this study were (1) to determine the basic firing properties of the neurons in the two nuclear groups of the amygdala and (2) to determine whether neurons in the two nuclear groups are engaged differentially by task events and/or by image content.

As suggested by cytoarchitectonic differences, the pallidostriatal neurons in the CM division showed higher firing rates and irregular firing patterns compared to the cortical type neurons in the BL division, which showed lower rates but more regular firing patterns.

One prominent aspect of firing properties documented here was the symmetrical pattern of excitatory and inhibitory re- sponses to various task events. The type of responses illustrated in Figure 2 were not rare occurrences; rather, the inhibitory responses made up one-quarter to one-third of the total number of responses of each type, with the exception of the inhibitory fixspot responses, which were rare (7\%). These sharp, symmetrical inhibitory and excitatory responses might result from feedback inhibition, prevalent in the BL nuclei (Smith et al., 1998) and/or reciprocal interactions between populations of inhibitory and excitatory neurons. The presence of electrically coupled networks of interneurons (Muller et al., 2005) could mediate precisely timed switches in the convergent but complementary inputs at the level of the recorded neurons. Further understanding of these interactions will require recording a larger number of neurons simultaneously from several nuclei of the amygdala.

Regardless of the mechanism(s) that give rise to these response properties, neurons in the amygdala can encode information along three different dimensions of their response pattern: (1) magnitude of firing rate change, (2) polarity (inhibitory vs excitatory), and (3) timing (phasic vs tonic). The neuron depicted in Figure 5 illustrates this point. Not only does it fire with a different polarity for objects and faces, it also discriminates faces based on the magnitude of its excitatory response. Similarly, the neuron shown in Figure 6, discriminates stimuli by firing with different timing properties (namely, the tonic response exclusive for objects) and firing rate magnitudes (different phasic firing rates for different monkey identities).

Neurons in the BL nuclei were more selective than CM neurons. The difference between the average selectivity indices was moderate but significant for the entire recorded population. Large differences in selectivity were not expected given the monosynaptic input from the selective neurons in the BL onto the neurons in the CM. The observed difference in selectivity can be attributed to a dimensionality reduction of the representation along the information flow from the BL nuclei to the CM nuclei. Image selectivity (differences of firing rate) and stimulus specificity (response to only a small fraction of stimuli) in the $\mathrm{BL}$ suggest that these representations are high-dimensional, commensurate with the diversity of stimuli received by the amygdala. Previous single-cell recordings in the monkey and human amygdala showed the existence of amygdala neurons that are highly selective for categories of images (faces) as well as for exemplars from a category (Gothard et al., 2007; Mormann et al., 2008; Viskontas et al., 2009). A high-dimensional representation is advantageous for associating specific stimuli with reward contingencies because it minimizes the overlap and possible interference between stimuli that might be perceptually similar but predict different outcomes. In the CM nuclei, the dimensionality of stimulus representation is likely to be reduced because the output of a large number of BL neurons converge onto a smaller number of CM neurons (Carlo et al. 2010). As a result of a dimensionality reduction, the representations in the CM nuclei can become commensurate with the relatively limited number of emotional expressions compared to the infinite number of stimuli that can cause them.

One aspect of the response properties of BL neurons that merits emphasis is their selectivity for images with no obvious emotional significance to the monkey (e.g., unfamiliar objects) (Fig. $3 B$ ). Neurons that were selective for faces sometimes responded more to nonface images (Fig. 6) that were possibly ambiguous to the viewer monkey. As predicted by Whalen et al. (1998), the amygdala might allocate increased resources to process images with ambiguous or uncertain content. This proposal has been confirmed experimentally in multiple species under multiple ex- 
perimental conditions (Critchley et al., 2001; Hsu et al., 2005; Herry et al., 2007; Platt and Huettel, 2008).

The observation that neurons in the CM nuclei respond preferentially to task events warrants several, mutually nonexclusive interpretations. One possibility is that the task-related responses (fix spot on, image on, image off) reflect instrumental and pavlovian associations with reward. The images themselves can act as reinforcement for correct gaze behavior. Indeed, the central nucleus has been shown to support conditioning (Killcross et al., 1997; Hall et al., 2001; Everitt et al., 2003; Wilensky et al., 2006). In monkeys, Belova et al. (2007) have shown that fix-spot responses can be regarded as the earliest predictors of reward. According to this scenario, the current results indicate that neurons in CM nuclei of the monkey amygdala are significantly more engaged in instrumental conditioning than neurons in the BL nuclei.

An alternative explanation for the higher propensity of the CM neurons to signal task events is the purported role of the amygdala in allocating attention to relevant stimuli (Kapp et al., 1994; Davis and Whalen 2001; Sander et al., 2003). Whether the fix-spot-on, image-on, and image-off events acquired relevance through conditioning or these events are intrinsically relevant for the ongoing behavior, it is clear that these events require some form of attention. The connection of the CM neurons to the nucleus basalis and the bed nucleus of the stria terminalis (Jones et al., 1976) might explain how increased activity in the central nucleus translates into increased attention and vigilance (Dringenberg and Vanderwolf, 1996).

A division of labor among the amygdala nuclei along the axes of attention/vigilance and emotion has been proposed previously and verified by nuclear-selective stimulation, lesion studies, as well as by the outcome of nuclear-specific pharmacological manipulations (for review, see Davis and Whalen, 2001; Pape and Paré, 2010). Although the present findings support the dual role of the amygdala in emotion and attention, several response characteristics of the recorded neurons (e.g., considerable overlap in the response properties of neurons from the two nuclear groups) suggest that this division of labor is not clear cut.

A new conceptual framework, the component process model of emotion (Sander et al., 2005), might also account for the observed response properties reported here, although it does not predict any nuclear specialization. According to this model, stimulus evaluation (appraisal) is the first component of several recursive processes that take place when an organism encounters a potentially important stimulus or event. Within this component, the first process establishes the relevance of the event, e.g., whether the stimulus is novel or familiar, whether is predicts negative or positive valence, and whether it serves the goals of the agent. In this framework, the appearance of the fix spot, the presentation of the image, and the content of the image engage the same relevance detector. Relevance in this framework encompasses multiple aspects of the stimuli: motivational (prediction of reward), social (faces versus nonface stimuli) (Fig. 5), and attentional (ambiguous images that might require further investigation) (Figs. 5, 6).

Although the present study lends support to several complementary views on the division of labor among the amygdala nuclei, it has several limitations. First, the localization of the recorded neurons to each nucleus was not precise enough to differentiate between the subdivisions of the CM and BL groups. We estimated localization errors up to a maximum of $0.5 \mathrm{~mm}$ in the dorsoventral axis and $1 \mathrm{~mm}$ in the mediolateral axis of the amygdala. Second, the sampling of the nuclei was unequal, which did not allow for a more detailed, nuclear-specific analysis. Third, the stimuli could not be classified in distinct and clear classes to determine whether faces and other well-defined classes of stimuli induce different temporal patterns of neural activity. Finally, extracellular recordings in awake monkeys can only partially capture the basic firing characteristics of neurons. The main classes of neurons in each nucleus of the amygdala have been characterized in great detail in rodents and cats (McDonald, 1992; Lambertz et al., 1995; Pape, 2005; Sosulina et al., 2006; Herry et al., 2007), but given species-specific differences (Pitkanen and Amaral, 1991; McDonald and Augustine, 1993) and the differences in experimental approach, the correspondence between classes of neurons described in the current study and the known neural architecture of the amygdala of lower species is hard to establish. An example of this challenge is the difficulty of identifying intercalated neurons in the monkey. We report a small number of neurons with distinct firing properties recorded from the expected location of the intercalated neurons; however, the regular firing patterns of these neurons do not agree with the firing patterns of anatomically confirmed, GABAergic intercalated neurons in rodents (Collins and Paré, 1999). Given the rarity of these neurons and their low, regular firing rates, they may belong to a subclass of cholinergic intercalated neurons (Nitecka and Frotscher, 1989).

Despite these limitations, these results indicate that even a simple image-viewing task engages the neurons of the amygdala to respond to multiple aspects of the ongoing behavior. Task events and the content of images appear to engage differentially neurons in the two major nuclear divisions of the amygdala. Future studies will further refine these nuclear specializations.

\section{References}

Adolphs R (2010) What does the amygdala contribute to social cognition? Ann N Y Acad Sciences 1191:42-61.

Adolphs R, Gosselin F, Buchanan TW, Tranel D, Schyns P, Damasio AR (2005) A mechanism for impaired fear recognition after amygdala damage. Nature 433:68-72

Amaral DG, Price JL (1984) Amygdalo-cortical projections in the monkey (Macaca fascicularis). J Comp Neurol 230:465-496.

Amaral DG, Price JL, Pitkanen A, Carmichael ST (1992) Anatomical organization of the primate amygdaloid complex. In: The amygdala: neurobiological aspects of emotion, memory, and mental dysfunction (Aggleton JP, ed), pp 1-66. New York: Wiley.

Belova MA, Paton JJ, Morrison SE, Salzman CD (2007) Expectation modulates neural responses to pleasant and aversive stimuli in primate amygdala. Neuron 55:970-984.

Carlo CN, Stefanacci L, Semendeferi K, Stevens CF (2010) Comparative analyses of the neuron numbers and volumes of the amygdaloid complex in old and new world primates. J Comp Neurol 518:1176-1198.

Collins DR, Paré D (1999) Spontaneous and evoked activity of intercalated amygdala neurons. Eur J Neurosci 11:3441-3448.

Critchley HD, Mathias CJ, Dolan RJ (2001) Neural activity in the human brain relating to uncertainty and arousal during anticipation. Neuron 29:537-545.

Davis M, Whalen PJ (2001) The amygdala: vigilance and emotion. Mol Psychiatry 6:13-34.

Dringenberg HC, Vanderwolf CH (1996) Cholinergic activation of the electrocorticogram: an amygdaloid activating system. Exp Brain Res 108:285-296.

Dumont EC, Martina M, Samson RD, Drolet G, Paré D (2002) Physiological properties of central amygdala neurons: species differences. Eur J Neurosci 15:545-552.

Eckhorn R, Thomas U (1993) A new method for the insertion of multiple microprobes into neural and muscular tissue, including fiber electrodes, fine wires, needles and microsensors. J Neurosci Methods 49:175-179.

Everitt BJ, Cardinal RN, Parkinson JA, Robbins TW (2003) Appetitive behavior: impact of amygdala-dependent mechanisms of emotional learning. Ann N Y Acad Sci 985:233-250. 
Gallagher M, Graham PW, Holland PC (1990) The amygdala central nucleus and appetitive pavlovian conditioning: lesions impair one class of conditioned behavior. J Neurosci 10:1906-1911.

Ghashghaei HT, Barbas H (2002) Pathways for emotion: interactions of prefrontal and anterior temporal pathways in the amygdala of the rhesus monkey. Neuroscience 115:1261-1279.

Gothard KM, Erickson CA, Amaral DG (2004) How do rhesus monkeys (Macaca mulatta) scan faces in a visual paired comparison task? Anim Cogn 7:25-36.

Gothard KM, Battaglia FP, Erickson CA, Spitler KM, Amaral DG (2007) Neural responses to facial expression and face identity in the monkey amygdala. J Neurophysiol 97:1671-1683.

Hall J, Parkinson JA, Connor TM, Dickinson A, Everitt BJ (2001) Involvement of the central nucleus of the amygdala and nucleus accumbens core in mediating pavlovian influences on instrumental behaviour. Eur J Neurosci 13:1984-1992.

Herry C, Bach DR, Esposito F, Di Salle F, Perrig WJ, Scheffler K, Luthi A, Seifritz E (2007) Processing of temporal unpredictability in human and animal amygdala. J Neurosci 27:5958-5966.

Holland PC, Gallagher M (1999) Amygdala circuitry in attentional and representational processes. Trends Cogn Sci 3:65-73.

Hsu M, Bhatt M, Adolphs R, Tranel D, Camerer CF (2005) Neural systems responding to degrees of uncertainty in human decision-making. Science 310:1680-1683

Jones EG, Burton H, Saper CB, Swanson LW (1976) Midbrain, diencephalic and cortical relationships of the basal nucleus of Meynert and associated structures in primates. J Comp Neurol 167:385-419.

Kaada BR (1951) Somato-motor, autonomic and electrocorticographic responses to electrical stimulation of rhinencephalic and other structures in primates, cat, and dog; a study of responses from the limbic, subcallosal, orbito-insular, piriform and temporal cortex, hippocampus-fornix and amygdala. Acta Physiol Scand Suppl 24:1-262.

Kapp BS, Gallagher M, Underwood MD, McNall CL, Whitehorn D (1982) Cardiovascular responses elicited by electrical stimulation of the amygdala central nucleus in the rabbit. Brain Res 234:251-262.

Kapp BS, Supple WF Jr, Whalen PJ (1994) Effects of electrical stimulation of the amygdaloid central nucleus on neocortical arousal in the rabbit. Behav Neurosci 108:81-93.

Killcross S, Robbins TW, Everitt BJ (1997) Different types of fearconditioned behaviour mediated by separate nuclei within amygdala. Nature 388:377-380.

Krettek JE, Price JL (1978) Amygdaloid projections to subcortical structures within the basal forebrain and brainstem in the rat and cat. J Comp Neurol 178:225-254.

Laine CM, Spitler KM, Mosher CP, Gothard KM (2009) Behavioral triggers of skin conductance responses and their neural correlates in the primate amygdala. J Neurophysiol 101:1749-1754.

Lambertz M, Schulz G, Langhorst P (1995) Cardiac rhythmic patterns in neuronal activity related to the firing rate of the neurons: II. Amygdala neurons of cats. J Auton Nerv Syst 51:165-173.

LeDoux J (2000) The amygdala and emotion: a view through fear. In: The amygdala, Ed 2 (Aggleton JP, ed), pp 289-310. Oxford, UK: Oxford UP.

McDonald AJ (1992) Cell types and intrinsic connections of the amygdala. In: The amygdala (Aggleton JP, ed), pp 67-96. New York: Wiley.

McDonald AJ (1998) Cortical pathways to the mammalian amygdala. Prog Neurobiol 55:257-332.

McDonald AJ, Augustine JR (1993) Localization of GABA-like immunoreactivity in the monkey amygdala. Neuroscience 52:281-294.

Mormann F, Kornblith S, Quiroga RQ, Kraskov A, Cerf M, Fried I, Koch C (2008) Latency and selectivity of single neurons indicate hierarchical processing in the human medial temporal lobe. J Neurosci 28:8865-8872.
Mountcastle VB, Reitboeck HJ, Poggio GF, Steinmetz MA (1991) Adaptation of the Reitboeck method of multiple microelectrode recording to the neocortex of the waking monkey. J Neurosci Methods 36:77-84.

Muller JF, Mascagni F, McDonald AJ (2005) Coupled networks of parvalbuminimmunoreactive interneurons in the rat basolateral amygdala. J Neurosci 25:7366-7376.

Nitecka L, Frotscher M (1989) Organization and synaptic interconnections of GABAergic and cholinergic elements in the rat amygdaloid nuclei: single- and double-immunolabeling studies. J Comp Neurol 279:470-488.

Pape HC (2005) GABAergic neurons: gate masters of the amygdala, mastered by dopamine. Neuron 48:877-879.

Pape HC, Paré D (2010) Plastic synaptic networks of the amygdala for the acquisition, expression, and extinction of conditioned fear. Physiol Rev 90:419-463.

Paré D, Gaudreau H (1996) Projection cells and interneurons of the lateral and basolateral amygdala: distinct firing patterns and differential relation to theta and delta rhythms in conscious cats. J Neurosci 16:3334-3350.

Paré D, Quirk GJ, Ledoux JE (2004) New vistas on amygdala networks in conditioned fear. J Neurophysiol 92:1-9.

Pascoe JP, Kapp BS (1985) Electrophysiological characteristics of amygdaloid central nucleus neurons in the awake rabbit. Brain Res Bull 14:331-338.

Pitkanen A, Amaral DG (1991) Demonstration of projections from the lateral nucleus to the basal nucleus of the amygdala: a PHA-L study in the monkey. Exp Brain Res 83:465-470.

Platt ML, Huettel SA (2008) Risky business: the neuroeconomics of decision making under uncertainty. Nat Neurosci 11:398-403.

Reis DJ, Ledoux JE (1987) Some central neural mechanisms governing resting and behaviorally coupled control of blood pressure. Circulation 76:I2-I9.

Roesch MR, Calu DJ, Esber GR, Schoenbaum G (2010) Neural correlates of variations in event processing during learning in basolateral amygdala. J Neurosci 30:2464-2471.

Sander D, Grafman J, Zalla T (2003) The human amygdala: an evolved system for relevance detection. Rev Neurosci 14:303-316.

Sander D, Grandjean D, Scherer KR (2005) A systems approach to appraisal mechanisms in emotion. Neural Netw 18:317-352.

Smith Y, Pare JF, Paré D (1998) Cat intraamygdaloid inhibitory network: ultrastructural organization of parvalbumin-immunoreactive elements. J Comp Neurol 391:164-179.

Sosulina L, Meis S, Seifert G, Steinhauser C, Pape HC (2006) Classification of projection neurons and interneurons in the rat lateral amygdala based upon cluster analysis. Mol Cell Neurosci 33:57-67.

Spitler KM, Gothard KM (2008) A removable silicone elastomer seal reduces granulation tissue growth and maintains the sterility of recording chambers for primate neurophysiology. J Neurosci Methods 169:23-26.

Stefanacci L, Amaral DG (2002) Some observations on cortical inputs to the macaque monkey amygdala: an anterograde tracing study. J Comp Neurol 451:301-323.

Viskontas IV, Quiroga RQ, Fried I (2009) Human medial temporal lobe neurons respond preferentially to personally relevant images. Proc Natl Acad Sci U S A 106:21329-21334.

Whalen PJ, Rauch SL, Etcoff NL, McInerney SC, Lee MB, Jenike MA (1998) Masked presentations of emotional facial expressions modulate amygdala activity without explicit knowledge. J Neurosci 18:411-418.

Wilensky AE, Schafe GE, Kristensen MP, LeDoux JE (2006) Rethinking the fear circuit: the central nucleus of the amygdala is required for the acquisition, consolidation, and expression of pavlovian fear conditioning. J Neurosci 26:12387-12396. 